\title{
Determination of the $\boldsymbol{k}$-Values of Copper-Gold Alloys with ED- and WD-EPMA - Results of an Inter-Laboratory Comparison
}

\author{
Vasile-Dan Hodoroaba ${ }^{1}$, Ryna B. Marinenko, ${ }^{2}$ Vanessa Rackwitz ${ }^{1}$, Wolfram Bremser ${ }^{3}$ and Wolfgang E. \\ S. Unger ${ }^{1}$ \\ 1. BAM Federal Institute for Materials Research and Testing, Division 6.8 Surface Analysis and \\ Interfacial Chemistry, 12200 Berlin, Germany. \\ 2. National Institute of Standards and Technology (NIST), Materials Measurement Science Division, \\ Microanalysis Research Group, Gaithersburg, MD 20899, USA. \\ 3. BAM Federal Institute for Materials Research and Testing, Division 1.4 Process Analytical \\ Technology, 12200 Berlin, Germany.
}

An inter-laboratory comparison organized as a pilot study (PS) has been performed under the umbrella of the Consultative Committee for Amount of Substance - Metrology in Chemistry (CCQM) / Surface Analysis Working Group (SAWG) with the objective to compare $k$-values measured with Electron Probe X-ray Microanalysis (EPMA) for copper-gold alloys. The $k$-value, defined as the ratio of the Xray photon intensity of an X-ray characteristic line of the unknown sample to the corresponding one in the reference material, is a rough estimate of the mass fraction of the respective element. Basically, the mass fractions can be calculated from the $k$-values after applying a matrix correction procedure, which includes empirical approaches, so that traceability gets lost. Due to its physical, measurable nature, the $k$-value has been selected in the present PS as the primary measurand. The mass fraction has been set as the secondary (derived) measurand. Both EDX and WDX have been employed. The WDX results and the overall comparability between the $k$-values obtained by EDX and WDX are still in progress.

Fourteen participants (see Acknowledgement) from eight national metrology institutes (NMI) and two companies submitted $k$-values measured with ED-EPMA. The NIST (NBS) microanalysis SRM 482, a set of four $\mathrm{Cu}-\mathrm{Au}$ alloys with different elemental compositions, was selected as the "unknown" samples. The reference standards used were the pure copper and gold that are included in the SRM 482. All participants used the same measurement protocols with specified measurement conditions and the same set of well-characterized samples and standards prepared by BAM. Using both $25 \mathrm{kV}$ and $5 \mathrm{kV}$ excitation potentials enables the analysis of the high energy lines, $\mathrm{Cu} \mathrm{K}$ and $\mathrm{Au} \mathrm{L}$, and the low energy lines $\mathrm{Cu} \mathrm{L}$ and $\mathrm{Au} \mathrm{M}$. Because of the sensitivity to surface morphology and contamination at $5 \mathrm{kV}$ special care was devoted to sample preparations.

The comparison of the results of the reported EDX $k$-values and the associated measurement uncertainties evaluated as recommended by ISO-GUM [2], appear in Fig. 1. One can conclude for this PS that the EDX inter-lab $k$-values are comparable. The primary contribution to the uncertainty has been identified to come from the measurement of the primary electron beam current.

Eight laboratories have reported WDX results for the $5 \mathrm{kV}$ and $25 \mathrm{kV}$ excitation potentials. As with the EDX analyses specific protocols were provided. The results of the analysis of just one of the alloys, $\mathrm{Au} 20-\mathrm{Cu} 80$, have been added to Figure 1. Five results were taken with instruments having a $40^{\circ}$ takeoff angle (TOA) while three results were from instruments with a $30^{\circ}$ TOA. No complete uncertainties values have been calculated because we are in process of determining them, hopefully for all laboratories. In Figure 1 only the single standard deviation for each WDX laboratory result is shown. 
Except for a few outliers, the WDX plots suggest that there is generally good agreement between the laboratories with $40^{\circ} \mathrm{TOA}$, but for the $30^{\circ} \mathrm{TOA}$ instruments, there is often disagreement between the three instruments. This diversity is true for the other alloys as well. The reason for the diversity is not presently known, but will be investigated.

\section{References:}

[1] V-D Hodoroaba, R Marinenko et al., Microsc. Microanal. 17 (suppl. S2) (2011), p. 856.

[2] ISO/IEC Guide 98-3:2008 Uncertainty of measurement Part 3: Guide to the expression of uncertainty in measurement (GUM:1995), Geneva: Internat. Org. Stds, 101 pp.

[3] All participants in this PS are thanked for their contribution: E Peldszus \& G Pelzel (AQura), I

Feldmann, G Oder \& S Benemann (BAM), R Terborg (Bruker Nano), C Hombourger \& M Outrequin (Cameca), J M Juarez (CENAM), I Popov \& I Kuselman (INPL), P McSwiggen (McSwiggen \& Associates), H Wang (NIM), E Steel \& J Small (NIST), L Adlem (NMISA), D Gohil (NPL), S Richter (RWTH Aachen) and L Lindman (SP).
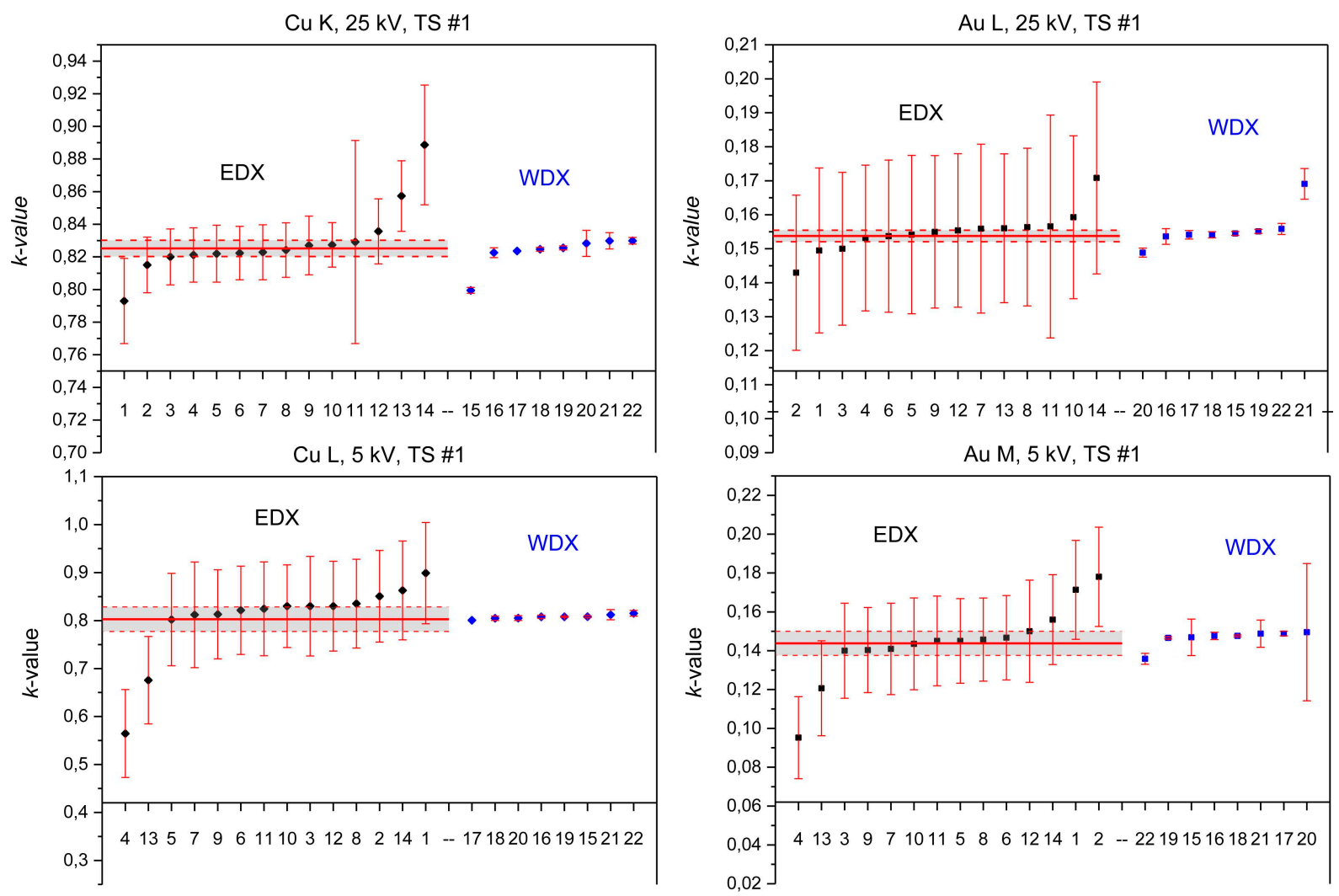

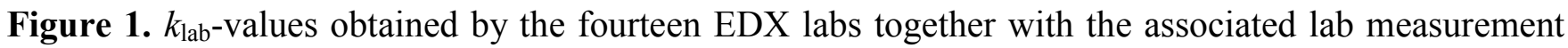
combined uncertainties, $u_{c}$, for $\mathrm{Cu} \mathrm{K}$ and $\mathrm{Au} \mathrm{L}$ at $25 \mathrm{kV}$ beam voltage (upper diagrams) and for $\mathrm{Cu} \mathrm{L}$ and $\mathrm{Au} \mathrm{M}$ at $5 \mathrm{kV}$ beam voltage (lower diagrams) - for the test sample TS \#1 (Au20-Cu80). The weighted mean $k$-value of the EDX inter-laboratory comparison, $k_{\text {inter-lab }}{ }^{\text {wt }}$ is also shown (continuous red lines) together with the associated uncertainty, $u_{\text {inter-lab }}$ wt (grey bands). All "EDX" uncertainties are single unexpanded values. The eight WDX $k_{\mathrm{lab}}$-values have been also added. Note that the uncertainty shown for the WDX laboratory results is "only" the single standard deviation. 